\title{
Dynamic evaluation of renal resistive index in normoalbuminuric patients with newly diagnosed hypertension or type 2 diabetes
}

\author{
R. M. Bruno • E. Daghini $\cdot$ L. Landini $\cdot$ D. Versari • \\ A. Salvati • E. Santini • I. Di Paco • A. Magagna • \\ S. Taddei • L. Ghiadoni $\cdot$ A. Solini
}

Received: 11 January 2011 / Accepted: 18 March 2011 / Published online: 16 April 2011

(C) Springer-Verlag 2011

\begin{abstract}
Aim/hypothesis Renal resistive index is a useful measure for quantifying alterations in renal blood flow. In the present study we evaluated resistive index at baseline and after vasodilation induced by nitroglycerine in normoalbuminuric patients with type 2 diabetes or essential hypertension, relating the values to indices of systemic vascular dysfunction.

Methods Newly diagnosed treatment-naïve type 2 diabetic $(n=32)$ and hypertensive patients $(n=49)$ were compared with 27 age- and sex-matched healthy controls. Renal resistive index was obtained by duplex ultrasound at baseline and after $25 \mu \mathrm{g}$ sublingual nitroglycerine. Endothelium-dependent (flow-mediated dilation) and -independent (response to nitroglycerine) vasodilation in the brachial artery was assessed by computerised edge detection system. Carotid-femoral pulse-wave velocity and augmentation index were assessed by applanation tonometry. Nitrotyrosine levels, an index of oxidative stress, were also measured.

Results Resistive index was higher in diabetic than in hypertensive patients and controls $(p<0.001)$, while changes in resistive index induced by nitroglycerine were lower in hypertensive patients compared with controls $(p<$ 0.01 ), and were further reduced in type 2 diabetic patients. Hypertensive and diabetic patients showed significantly
\end{abstract}

R. M. Bruno and E. Daghini contributed equally to this study.

R. M. Bruno • E. Daghini $\cdot$ L. Landini $\cdot$ D. Versari $\cdot$ A. Salvati

E. Santini $\cdot$ I. Di Paco $\cdot$ A. Magagna $\cdot$ S. Taddei $\cdot$

L. Ghiadoni $(\bowtie) \cdot$ A. Solini

Department of Internal Medicine, University of Pisa,

Via Roma 67,

56100 Pisa, Italy

e-mail: 1.ghiadoni@med.unipi.it increased arterial stiffness, nitrotyrosine levels and reduced endothelial function than controls $(p<0.05)$. Changes in resistive index induced by nitroglycerine were independently related to serum glucose, reactive hyperaemia and aortic pulse-wave velocity in the overall population.

Conclusions/interpretation These results support the dynamic evaluation of renal resistive index as an early detector of renal vascular alterations in the presence of type 2 diabetes and hypertension, even before the onset of microalbuminuria.

Keywords Arterial stiffness - Endothelial function · Hypertension - Renal resistive index · Type 2 diabetes

$\begin{array}{ll}\text { Abbreviations } \\ \text { AIx } & \text { Augmentation index } \\ \text { bpm } & \text { Beats per min } \\ \text { DRIN } & \text { Dynamic resistive index } \\ \text { EH } & \text { Essential hypertensive patients } \\ \text { FMD } & \text { Flow-mediated dilation } \\ \text { GTN } & \text { Glyceryl trinitrate } \\ \text { PP } & \text { Pulse pressure } \\ \text { PWV } & \text { Pulse-wave velocity } \\ \text { RI } & \text { Renal resistive index } \\ \text { UACR } & \text { Urine albumin-to-creatinine ratio }\end{array}$

\section{Introduction}

Early diagnosis of target organ damage has a relevant role for patients at high cardiovascular risk, as both the presence and the regression of initial alterations, including left ventricle hypertrophy, intima-media thickness and microalbuminuria, are independent determinants of cardiovascu- 
lar prognosis [1]. With regard to large arteries, endothelial dysfunction and arterial stiffness have been proved to be indices of early vascular pathological changes, which might lead to end-organ dysfunction [2-4]. The relevance of these indicators is growing, as aortic stiffness-measured as aortic pulse-wave velocity (PWV) [2] - has now entered into international guidelines for the detection of hypertensive organ damage [1]. Microalbuminuria is recognised as a clinical marker of the first stages of nephropathy in type 1 and 2 diabetes $[5,6]$, whereas in essential hypertension it is mainly considered an indicator of cardiovascular risk $[1,5]$. However, microalbuminuria identifies an already established glomerular damage, which is likely to be preceded by earlier structural and functional alterations undetectable to date, unless by kidney biopsy [7-9]. Moreover, a variable percentage of type 2 diabetic patients show an impairment of GFR even in the presence of normal albumin excretion [10]. Thus, new reliable markers of early renal dysfunction, specifically linked to vascular renal impairment, are needed in order to better identify the mechanisms responsible and the ideal timing of treatment, aiming to delay the onset and/ or slow the progression of renal damage.

Renal resistive index (RI) measured by duplex ultrasound is a useful measure for quantifying the alterations in intraparenchymal renal circulation that may occur during the course of chronic renal disease. RI is tightly related to renal arteriolosclerosis, as demonstrated by bioptic studies [11], and represents an integrated index of arterial compliance, pulsatility and downstream microvascular impedance $[12,13]$. High RI has a negative prognostic value in diabetic patients in terms of progression of renal disease [14] and in hypertensive patients with renal artery stenosis for the success of percutaneous revascularisation [15]. However, some limitations must be considered concerning the use of RI in the early phases of nephropathy. First, reference values of RI in the general population have not been unanimously defined and validated; second, RI is critically influenced by the ageing process [16]; third, in the presence of normal renal function, the narrow range of RI values might not be able to highlight initial renal microvascular alterations. Therefore, the use of a pharmacological stimulus inducing vasodilation applied to RI measurements (dynamic RI [DRIN]) might increase the discriminating power of duplex ultrasound, configuring a method potentially able to unmask renal abnormalities even in the early phases.

A reduced renal vasodilation with nitroglycerine has been demonstrated in patients with overt diabetic nephropathy [17]; whether an impaired renal vasodilation is present even before the onset of microalbuminuria in patients with type 2 diabetes or with other cardiovascular risk factors, and its potential relation to markers of systemic vascular dysfunction, is still unknown.
The aim of the present study was to evaluate RI in basal conditions and under pharmacological vasodilatory stimulus in newly diagnosed treatment-naive type 2 diabetic patients with normal urinary albumin excretion and GFR, comparing them with a group of patients with essential hypertension, in order to investigate whether a reduced DRIN could be a specific feature of diabetes. As a secondary aim, this study investigated the possible association of DRIN with established indices of systemic vascular damage, such as endothelial function and arterial stiffness, and increased oxidative stress.

\section{Methods}

Patients In the present study 108 individuals were included: 32 newly diagnosed ( $<3$ months), treatment-naive type 2 diabetic patients, defined according to the ADA criteria [18]; 49 never-treated essential hypertensive patients (EH); and 27 healthy participants, serving as controls. Type 2 diabetic and EH participants were recruited consecutively from January to June 2009 in the metabolism and hypertension outpatient clinic at the University Hospital, Pisa, Italy, while the controls were enrolled on a voluntary basis. Inclusion criteria were: age between 40 and 70 years; written informed consent; diagnosis of essential hypertension (for the EH group) or type 2 diabetes (for the diabetic group) within the previous 6 months, according to current guidelines $[1,18]$. Criteria of exclusion were: previous or current chronic treatment with anti-hypertensive or glucoselowering medications; reduced renal function (estimated GFR $<60 \mathrm{ml} \mathrm{min}^{-1} 1.73 \mathrm{~m}^{-2}$ ); micro- or macroalbuminuria in any measurement in the previous 6 months preceding the study; clinical or laboratory signs of inflammatory diseases or other major comorbidities; history of cardiovascular disease; and secondary hypertension. Type 2 diabetic patients were all free of diabetic retinopathy. In accordance with institutional guidelines, the protocol was approved by the local ethics committee and all patients gave written consent.

Study design and biochemical determinations Patients were asked not to eat, and to avoid caffeine-containing beverages, alcohol, strenuous exercise and smoking for the $12 \mathrm{~h}$ prior to the experiment. Measurements were performed in the morning with participants supine and at rest in a quiet air-conditioned room $\left(22-24^{\circ} \mathrm{C}\right)$. Blood was collected from all patients to measure, by standard techniques, fasting glucose and $\mathrm{HbA}_{1 \mathrm{c}}$, serum creatinine, total cholesterol, HDL-cholesterol and triacylglycerols. GFR was estimated using the Chronic Kidney Disease Epidemiology Collaboration (CKD-EPI) formula [19]. Urinary albumin excretion was evaluated as the urine albumin-to-creatinine ratio 
(UACR) of spot morning urine samples collected on three different days during the month preceding the study. Plasma nitrotyrosine was analysed by using the Nitrotyrosine ELISA Test Kit (Cell Sciences, Canton, MA, USA) according to the manufacturer's instructions (sensitivity= $2 \mathrm{nmol} / \mathrm{l}$ ). Clinic BP (mean of at least two measurements in 5 min by an automatic sphygmomanometer [OMRON M4; Omron Corporation, Kyoto, Japan]) and heart rate were measured at the beginning and at the end of the study. The timeline of the study protocol is illustrated in Fig. 1.

Baseline and dynamic RI The duplex ultrasound intraparenchymal renal scan was performed by a single trained operator using an ultrasound machine (MyLab 25, Esaote, Florence, Italy) with a high-resolution multifrequency Convex probe $(2.5-4.5 \mathrm{MHz})$. Three velocimetric measurements of the interlobar renal arteries adjacent to medullary pyramids were obtained with a translumbar or anterior approach. RI was calculated in both kidneys according to the formula: (systolic peak velocity - end diastolic velocity)/systolic peak velocity. RI measurement was obtained at baseline and 5 min after pharmacological stimulus with a low dose $(25 \mu \mathrm{g})$ administration of sublingual glyceryl trinitrate (GTN; Fig. 1), a dose already shown to have no influence on BP or heart rate [20]. DRIN was calculated as absolute percentage change from baseline in response to GTN. The intra-observer coefficient of variation was $2.1 \%$ for RI and $11.6 \%$ for DRIN measurements.

Endothelium-dependent and -independent vasodilation in the brachial artery Endothelial function was assessed as vasodilation of the brachial artery in response to postischaemic reactive hyperaemia (flow-mediated dilation [FMD]) as previously described by Virdis et al. and Ghiadoni et al. [3, 20]. Briefly, a paediatric cuff was positioned around the right forearm $2 \mathrm{~cm}$ below the elbow and the right brachial artery was located and scanned longitudinally between 5 and $10 \mathrm{~cm}$ above the elbow using a $10 \mathrm{MHz}$ linear array transducer (MyLab 25, Esaote). The transducer was held at the same point throughout the scan by a stereotactic clamp to ensure consistency of the image. After 1 min baseline recording, the cuff was inflated for $5 \mathrm{~min}$ at $300 \mathrm{mmHg}$ and then deflated to induce reactive hyperaemia (Fig. 1). Endothelium-independent vasodilation was obtained by the sublingual administration of $25 \mu \mathrm{g}$ GTN $[3,20]$. Brachial artery diameter measurements were performed by a computerised edge detection system [21]. FMD and response to GTN were calculated as the maximal percentage increase in diameter above baseline (mean of measures obtained during the first minute). The coefficient of variation of FMD in our laboratory is 14\% [21].

The volume of blood flow was calculated by multiplying duplex flow velocity (corrected for the angle) by heart rate and vessel cross-sectional area $\left(\pi r^{2}\right)$. Flow velocity was measured at baseline and within $15 \mathrm{~s}$ after cuff release. Reactive hyperaemia was calculated as the maximum percentage increase in flow after cuff release compared with baseline flow.

Arterial tonometry Arterial tonometry (SphygmoCor, AtCor Medical, Sydney, NSW, Australia) was performed according to the international recommendations [2]. A handheld probe was placed on the selected artery and 10 to 15 subsequent images were recorded. Central pressure was derived from radial pressure waveform by means of a validated transfer function on three successive measurements. Augmented pressure was calculated as the difference between the second and the first systolic peaks, and augmentation index (AIx) was calculated as the ratio between augmented pressure and pulse pressure (PP) and normalised at a heart rate of 75 beats per minute (bpm).
Fig. 1 Timeline of the study protocol. The dashed lines show the periods of real-time brachial artery measurement. $\mathrm{VD}$, vasodilation

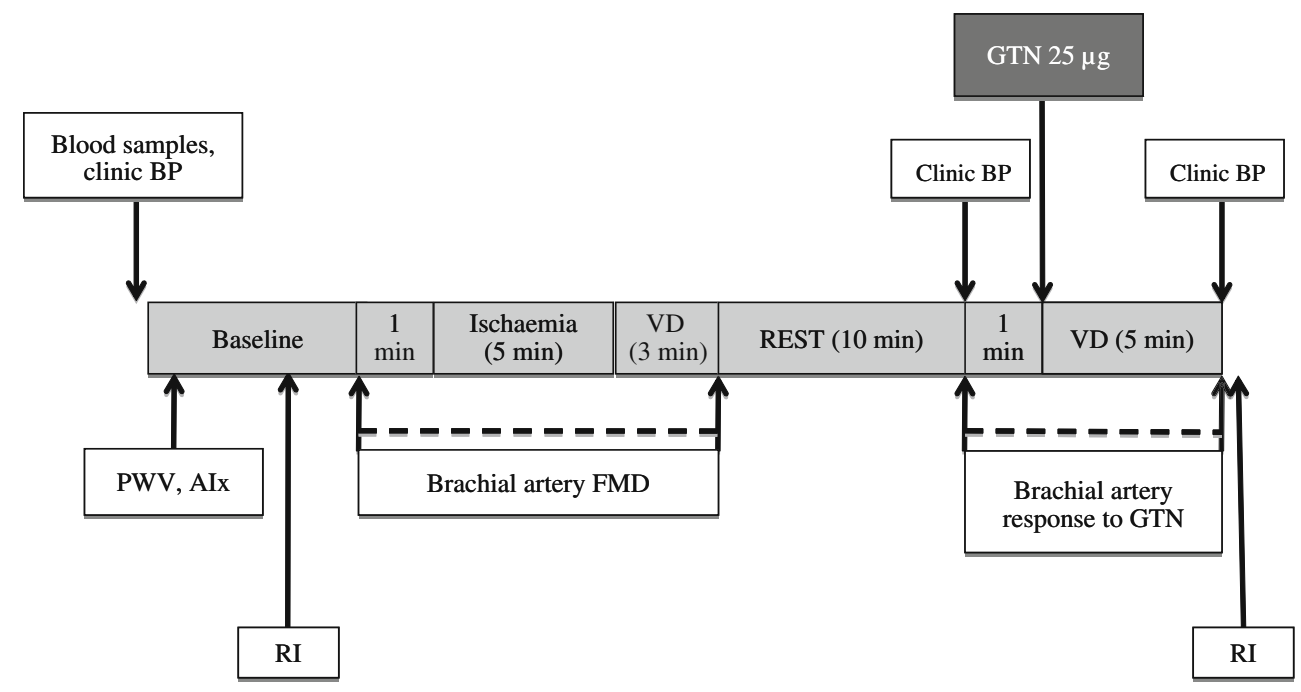


Aortic PWV was assessed with the same device, recording waveforms at the femoral and carotid sites, sequentially. PWV was calculated as the ratio of the surface distance between the two recording sites and wave-transit time. Variation coefficients for repeated measurement of AIx and PWV in our laboratory were $14 \%$ and $13 \%$, respectively [22].

Statistical analysis Statistical analysis was performed using NCSS 2004 (NCSS, Kaysville, UT, USA). The results were expressed as mean $\pm \mathrm{SD}$. Differences among groups were analysed using ANOVA and Fisher least-significant difference post hoc analysis for normally distributed variables, or Kruskal-Wallis $Z$ test for non-normally distributed variables; categorical variables were analysed by $\chi^{2}$ test. Multiple linear regression was applied to build a model to identify the determinants of DRIN and RI. Among clinical factors, age, creatinine, fasting glucose and PP were included, then adding those vascular variables that were significant in the univariate analysis. For both DRIN and RI two models were performed: model 1 , including brachial PP, and model 2, including aortic PP. Non-normally distributed variables were log-transformed for this analysis. A value of $p<0.05$ was considered significant.

\section{Results}

Clinical variables of the study groups are summarised in Table 1. Diabetic patients had significantly higher BMI compared with the other groups. UACR, even within the normal range, was higher in participants with type 2 diabetes than in control and EH participants, while serum creatinine, uric acid and estimated GFR were not significantly different between any groups (Table 1). HDLcholesterol and triacylglycerols were lower and higher in the $\mathrm{EH}$ and diabetic groups, respectively, compared with the controls. Neither the systolic nor the diastolic BP or heart rate were significantly modified by GTN administration in the overall population $(-0.6 \pm 3.0 \%,-0.8 \pm 5.8 \%$ and $-0.9 \pm 7.9 \%$ respectively).

RI values were significantly higher in participants with type 2 diabetes compared with control and $\mathrm{EH}$ participants $(0.65 \pm 0.06,0.59 \pm 0.05$ and $0.58 \pm 0.05$ respectively), while those participants with EH showed values superimposable to controls (Fig. 2a). Only a minority of individuals had an RI $>0.70$ ( 8 out of 108). DRIN was significantly reduced in both groups of patients (diabetic group 7.1 $\pm 6.1 \%$; EH 9.0 \pm $5.2 \%)$ compared with controls $(11.1 \pm 6.9 \%)$; of note, participants with type 2 diabetes showed a reduced DRIN compared with that of EH participants (Fig. 2b).

Systemic vascular variables in the three study groups are summarised in Table 2. FMD was reduced in EH and in type 2 diabetes patients compared with controls, while the endothelium-independent brachial vasodilation was not significantly different among the three groups. Reactive hyperaemia was reduced in type 2 diabetes compared with the controls and EH participants. Aortic PWV was higher in $\mathrm{EH}$ and in diabetic patients than in controls, whereas the timing of the reflected wave was significantly shortened. AIx was not significantly different within the three groups.

To test whether these differences might be related to an increased degree of oxidative stress in type 2 diabetes, we measured circulating levels of nitrotyrosine, which were significantly increased in type 2 diabetes and EH $(0.56 \pm$ 0.10 and $0.43 \pm 0.06 \mu \mathrm{mol} / \mathrm{l}$, respectively) compared with controls $(0.39 \pm 0.12 \mu \mathrm{mol} / \mathrm{l})$, and also significantly higher in type 2 diabetes than in $\mathrm{EH}(p<0.05)$.

Neither RI nor DRIN was affected by smoking status or $\operatorname{sex}(p=\mathrm{ns}$ for both). A univariate correlation analysis (Table 3) and a multiple regression analysis (Table 4) were then performed in order to identify the independent predictors of DRIN and RI.

In the univariate analysis on the overall population, DRIN was significantly correlated to both clinical (age, fasting glucose, brachial and aortic systolic BP and PP) and vascular factors (baseline RI, AIx, PWV, brachial response to GTN, reactive hyperaemia), as illustrated in Table 3. In the multiple regression analysis (Table 4), DRIN was independently related to fasting glucose, PWV and reactive hyperaemia in both models 1 and 2 including, respectively, brachial (full model $\left.r^{2}=0.41\right)$ and aortic PP $\left(r^{2}=0.39\right)$. RI was significantly correlated to both clinical factors (age, fasting glucose, creatinine, brachial PP, aortic systolic BP and PP) and vascular factors (timing of the reflected wave, PWV, FMD, reactive hyperaemia; Table 3). However, when multiple regression analysis was performed (Table 4), RI remained independently related only to fasting glucose, creatinine and aortic PP in model 2. This relationship was less close in model 1 , which included brachial instead of aortic PP.

When univariate analysis was performed in the type 2 diabetes group, DRIN was correlated with aortic PP ( $r=$ $-0.54, p<0.01)$, aortic systolic BP $(r=-0.40, p=0.003)$ and $\mathrm{HbA}_{1 \mathrm{c}}(r=-0.47, p<0.01)$, while for RI the correlation was present with brachial $(r=0.44, p=0.02)$ and aortic PP $(r=$ $0.52, p=0.004)$ and brachial systolic BP $(r=0.38, p=0.04)$. DRIN was not related to any of the other variables considered, including baseline RI and UACR.

In the EH group, DRIN correlated with brachial $(r=$ $-0.43, p=0.004)$ and aortic systolic BP $(r=-0.50, p<$ $0.001)$, brachial $(r=-0.31, p=0.04)$ and aortic PP $(r=-0.54$, $p<0.001)$, PWV $(r=-0.40, p<0.01)$, timing of the reflected wave $(p=0.32, r=0.04)$, AIx $(r=-0.51, p<0.001)$; RI was related to age $(r=0.31, p=0.04)$, brachial $(r=0.40, p=$ $0.007)$ and aortic systolic BP $(r=0.44, p=0.004)$, aortic PP 
Table 1 Clinical characteristics of the study population

\begin{tabular}{|c|c|c|c|c|}
\hline Characteristic & Controls $(n=27)$ & $\mathrm{EH}(n=49)$ & Type 2 diabetes $(n=32)$ & $p$ value \\
\hline Age (years) & $51.0 \pm 7.1$ & $51.8 \pm 8.8$ & $55.3 \pm 9.6$ & 0.12 \\
\hline Males, $n(\%)$ & $15(56)$ & $34(69)$ & $19(59)$ & 0.43 \\
\hline Smokers, $n(\%)$ & $5(19)$ & $11(22)$ & $6(19)$ & 0.89 \\
\hline Body mass index $\left(\mathrm{kg} / \mathrm{m}^{2}\right)$ & $26.1 \pm 4.1^{\dagger}$ & $27.2 \pm 4.3^{\dagger}$ & $31.0 \pm 6.7^{* *}$ & 0.003 \\
\hline Heart rate $(\mathrm{bpm})$ & $67.8 \pm 11.4$ & $66.6 \pm 10.5$ & $72.0 \pm 12.9$ & 0.12 \\
\hline Fasting glucose $(\mathrm{mmol} / \mathrm{l})$ & $5.1(4.4-5.4)^{\dagger}$ & $4.9(4.8-5.4)^{\dagger}$ & $7.1(7.0-10.2)^{* \ddagger}$ & $<0.001$ \\
\hline $\mathrm{HbA}_{1 \mathrm{c}}(\%)$ & $5.7 \pm 0.8^{\dagger}$ & $5.6 \pm 0.6^{\dagger}$ & $7.3 \pm 1.6^{* \pm}$ & $<0.001$ \\
\hline Serum creatinine $(\mu \mathrm{mol} / 1)$ & $79.6 \pm 14.1$ & $78.7 \pm 16.8$ & $78.7 \pm 15.1$ & 0.98 \\
\hline Estimated GFR $\left(\mathrm{ml} \mathrm{min} \min ^{-1} 1.73^{-2}\right)$ & $89.4 \pm 9.8$ & $92.1 \pm 14.6$ & $86.5 \pm 16.1$ & 0.36 \\
\hline UACR (mg/g) & $1.5(0.3-3.0)^{\dagger}$ & $1.5(0.5-7.2)^{\dagger}$ & $7.8(2.9-10.8)^{* \ddagger}$ & 0.01 \\
\hline Total cholesterol $(\mathrm{mmol} / \mathrm{l})$ & $5.7 \pm 0.9$ & $5.5 \pm 1.1$ & $5.9 \pm 1.4$ & 0.65 \\
\hline LDL-cholesterol (mmol/l) & $3.6 \pm 0.6$ & $3.6 \pm 1.0$ & $3.4 \pm 1.5$ & 0.80 \\
\hline HDL-cholesterol (mmol/l) & $1.5 \pm 0.5^{\dagger *}$ & $1.2 \pm 0.3^{*}$ & $1.2 \pm 0.3^{*}$ & $<0.001$ \\
\hline Triacylglycerols (mmol/l) & $0.9(0.8-1.3)^{\dagger+}$ & $1.8(1.2-2.4)^{*}$ & $2.3(1.5-2.9)^{*}$ & $<0.001$ \\
\hline Uric acid $(\mu \mathrm{mol} / \mathrm{l})$ & $280 \pm 54$ & $333 \pm 101$ & $297 \pm 71$ & 0.138 \\
\hline Brachial systolic BP $(\mathrm{mmHg})$ & $130.3 \pm 8.0^{\dagger \neq}$ & $145.6 \pm 10.3^{* \dagger}$ & $137.5 \pm 12.6^{* \pm}$ & $<0.001$ \\
\hline Brachial diastolic BP $(\mathrm{mmHg})$ & $78.5 \pm 6.1^{\dagger \ddagger}$ & $86.7 \pm 9.6^{* \dagger}$ & $78.0 \pm 8.3^{*}$ & $<0.001$ \\
\hline Brachial PP (mmHg) & $51.8 \pm 7.6^{\dagger *}$ & $58.9 \pm 13.1^{*}$ & $59.9 \pm 14.5^{*}$ & 0.03 \\
\hline Mean BP (mmHg) & $97.2 \pm 6.9^{\ddagger}$ & $103.4 \pm 16.7^{* \dagger}$ & $99.2 \pm 9.8^{*}$ & $<0.001$ \\
\hline Aortic systolic BP (mmHg) & $120.5 \pm 9.1^{\ddagger}$ & $133.3 \pm 12.4^{* \dagger}$ & $125.2 \pm 15.6^{\ddagger}$ & $<0.001$ \\
\hline Aortic PP (mmHg) & $41.5 \pm 8.1^{\dagger *}$ & $49.0 \pm 15.6^{*}$ & $47.6 \pm 15.5^{*}$ & $<0.001$ \\
\hline
\end{tabular}

Data are mean $\pm \mathrm{SD}$ or median $(25-75 \%)$

$p$ values are for trend (ANOVA) or $\chi^{2}$

${ }^{*} p<0.05$ vs controls; ${ }^{\dagger} p<0.05$ vs patients with type 2 diabetes; ${ }^{*} p<0.05$ vs EH

$(r=0.42, p=0.008)$ and timing of the reflected wave $(r=0.34$, $p=0.03)$.

In the overall population, nitrotyrosine was significantly related to both RI and DRIN (Table 3). However significance was lost for both variables after inclusion of clinical factors (age, PP, creatinine, fasting glucose) in the multiple regression model.

\section{Discussion}

The present study demonstrates that drug-induced renal vasodilation is impaired in newly diagnosed never-treated type 2 diabetic and hypertensive patients without clinically evident nephropathy. Moreover, it suggests that the estimation of the DRIN by duplex ultrasonography, a relatively
Fig. 2 Dot-plots representing baseline RI (a) and DRIN (b) in the three study groups. ${ }^{*} p<$ 0.05. C, controls; T2DM, participants with type 2 diabetes a

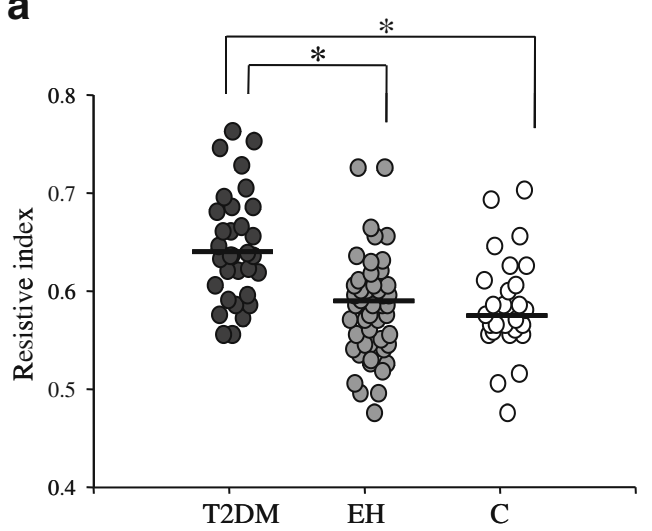

b

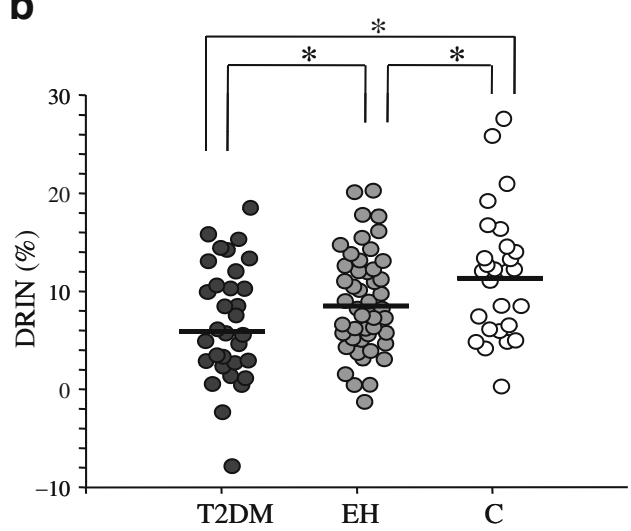


Table 2 Systemic vascular variables in the three study groups

\begin{tabular}{|c|c|c|c|c|}
\hline Variable & Control $(n=27)$ & $\mathrm{EH}(n=49)$ & Type 2 diabetes $(n=32)$ & $p$ value \\
\hline Brachial artery diameter $(\mathrm{mm})$ & $4.2 \pm 0.9$ & $4.2 \pm 0.9$ & $4.4 \pm 0.6$ & 0.64 \\
\hline Reactive hyperaemia (\%) & $674 \pm 290^{\dagger}$ & $532 \pm 254^{\dagger}$ & $362 \pm 223^{* *}$ & 0.001 \\
\hline FMD $(\%)$ & $6.7 \pm 3.3^{\dagger \dagger}$ & $4.9 \pm 2.4^{*}$ & $3.9 \pm 1.7^{*}$ & $<0.001$ \\
\hline Brachial artery response to GTN (\%) & $6.1 \pm 3.7$ & $6.4 \pm 3.0$ & $5.8 \pm 2.3$ & 0.65 \\
\hline AIx & $21.1 \pm 12.5$ & $27.0 \pm 22.1$ & $24.6 \pm 14.1$ & 0.33 \\
\hline Timing of the reflected wave (ms) & $147 \pm 23^{\dagger}$ & $138 \pm 18$ & $130 \pm 18^{*}$ & $<0.001$ \\
\hline Aortic PWV (m/s) & $7.5 \pm 1.1^{\dagger \dagger}$ & $8.2 \pm 1.7^{*}$ & $8.6 \pm 1.8^{*}$ & 0.004 \\
\hline
\end{tabular}

Data are mean $\pm \mathrm{SD}$

$p$ values are for trend (ANOVA)

${ }^{*} p<0.05$ vs $\mathrm{C} ;{ }^{\dagger} p<0.05$ vs type 2 diabetes; ${ }^{*} p<0.05$ vs EH

simple and low-cost technique, is able to identify subtle alterations in intra-renal vasculature even before albumin excretion has passed the cut-off for the definition of microalbuminuria. DRIN correlates with variables of
Table 3 Clinical and vascular factors affecting DRIN and baseline RI in the overall population - univariate analysis

\begin{tabular}{|c|c|c|c|c|}
\hline \multirow[t]{2}{*}{ Factor } & \multicolumn{2}{|l|}{ DRIN } & \multicolumn{2}{|l|}{ RI } \\
\hline & $r$ & $p$ value & $r$ & $p$ value \\
\hline \multicolumn{5}{|l|}{ Clinical } \\
\hline Age & -0.351 & $<0.001$ & 0.386 & $<0.001$ \\
\hline Ln (BMI) & 0.023 & 0.819 & 0.024 & 0.811 \\
\hline Ln (fasting glucose) & -0.122 & 0.296 & 0.420 & $<0.001$ \\
\hline $\operatorname{Ln}\left(\mathrm{HbA}_{1 \mathrm{c}}\right)$ & -0.040 & 0.849 & 0.173 & 0.408 \\
\hline Serum creatinine & 0.043 & 0.724 & -0.308 & 0.008 \\
\hline Estimated GFR & 0.191 & 0.114 & 0.053 & 0.657 \\
\hline Ln (UACR) & 0.192 & 0.168 & 0.227 & 0.083 \\
\hline Total cholesterol & 0.010 & 0.928 & 0.081 & 0.499 \\
\hline LDL-cholesterol & -0.160 & 0.197 & 0.001 & 0.990 \\
\hline HDL-cholesterol & -0.058 & 0.635 & -0.019 & 0.871 \\
\hline Ln (triacylglycerols) & -0.039 & 0.744 & 0.087 & 0.467 \\
\hline Uric acid & 0.051 & 0.729 & -0.154 & 0.292 \\
\hline Heart rate & 0.017 & 0.867 & -0.036 & 0.726 \\
\hline Brachial systolic BP & -0.208 & 0.042 & 0.167 & 0.099 \\
\hline Brachial diastolic BP & 0.047 & 0.646 & -0.181 & 0.074 \\
\hline Brachial PP & -0.246 & 0.016 & 0.388 & $<0.001$ \\
\hline Mean BP & -0.107 & 0.312 & -0.025 & 0.805 \\
\hline Aortic systolic BP & -0.357 & $<0.001$ & 0.219 & 0.033 \\
\hline Aortic PP & -0.490 & $<0.001$ & 0.419 & $<0.001$ \\
\hline \multicolumn{5}{|l|}{ Vascular factors } \\
\hline Brachial diameter at baseline & -0.064 & 0.553 & -0.035 & 0.739 \\
\hline Reactive hyperaemia & 0.318 & 0.009 & -0.276 & 0.025 \\
\hline FMD & 0.173 & 0.104 & -0.298 & 0.004 \\
\hline Brachial response to GTN & 0.242 & 0.023 & 0.024 & 0.819 \\
\hline AIx & -0.357 & $<0.001$ & 0.174 & 0.096 \\
\hline Timing of the reflected wave & 0.163 & 0.121 & -0.253 & 0.013 \\
\hline PWV & -0.373 & $<0.001$ & 0.260 & 0.018 \\
\hline Nitrotyrosine & -0.241 & 0.020 & 0.421 & $<0.001$ \\
\hline RI & -0.279 & 0.005 & - & - \\
\hline
\end{tabular}


Table 4 Clinical and vascular factors affecting DRIN and baseline RI in the overall population-multivariate analysis

\begin{tabular}{|c|c|c|c|c|c|c|c|c|}
\hline \multirow[t]{3}{*}{ Factor } & \multicolumn{4}{|l|}{ DRIN } & \multicolumn{4}{|l|}{ RI } \\
\hline & \multicolumn{2}{|c|}{ Model 1} & \multicolumn{2}{|c|}{ Model 2} & \multicolumn{2}{|c|}{ Model 1} & \multicolumn{2}{|c|}{ Model 2} \\
\hline & $r^{2}$ & $p$ value & $r^{2}$ & $p$ value & $r^{2}$ & $p$ value & $r^{2}$ & $p$ value \\
\hline \multicolumn{9}{|l|}{ Clinical } \\
\hline Age & 0.026 & 0.111 & 0.026 & 0.165 & 0.065 & 0.310 & 0.063 & 0.463 \\
\hline Serum creatinine & 0.025 & 0.079 & 0.025 & 0.107 & 0.182 & 0.045 & 0.180 & 0.048 \\
\hline Ln (serum glucose) & 0.124 & 0.004 & 0.103 & 0.009 & 0.061 & 0.088 & 0.097 & 0.025 \\
\hline Brachial PP & 0.007 & 0.321 & - & - & 0.107 & 0.053 & - & - \\
\hline Aortic PP & - & - & 0.042 & 0.810 & - & - & 0.076 & 0.036 \\
\hline \multicolumn{9}{|l|}{ Vascular } \\
\hline Reactive hyperaemia & 0.097 & 0.031 & 0.090 & 0.040 & 0.001 & 0.911 & 0.001 & 0.973 \\
\hline Brachial response to GTN & 0.022 & 0.742 & 0.016 & 0.809 & - & - & - & - \\
\hline AIx & 0.027 & 0.828 & 0.028 & 0.948 & - & - & - & - \\
\hline PWV & 0.070 & 0.006 & 0.045 & 0.018 & 0.001 & 0.730 & 0.003 & 0.704 \\
\hline RI & 0.006 & 0.224 & 0.010 & 0.462 & & & & \\
\hline Flow-mediated dilation & - & - & - & - & 0.001 & 0.812 & 0.008 & 0.869 \\
\hline Timing of the reflected wave & - & - & - & - & 0.003 & 0.855 & 0.001 & 0.673 \\
\hline
\end{tabular}

vascular function and arterial stiffness, beyond the effect of classic cardiovascular risk factors, suggesting parallel damage progression in different vascular target districts. In particular, DRIN is related to metabolic and BP control in the diabetic subgroup, and with arterial stiffness and wave reflections in hypertensive individuals, confirming that different mechanisms are involved in the development of renal damage in the early stages of the two diseases.

Clinical markers of renal dysfunction, such as GFR and urinary albumin excretion, are extensively used as indicators of renal damage in hypertensive [1] and diabetic patients [23]; however, indicators of early impairment of kidney function during the clinical course of chronic nephropathies are required, in particular to distinguish parenchymal from microvascular abnormalities. In this view, duplex evaluation of RI has recently been implemented in order to better detect early renal vascular alterations. A mean renal RI of around 0.60 in individuals without pre-existing renal diseases [24] and a cut-off value of 0.70 for the upper threshold of normality $[16,25,26]$ have been suggested. An elevated RI was found to correlate with left ventricular hypertrophy and carotid intima-media thickening [27-29], suggesting this index as a marker of systemic structural organ damage. However, in the present study only a small percentage of patients had RI values above the 0.70 cut-off. Moreover, in hypertensive patients RI values were not different from those in normal participants, suggesting that this duplex variable does not discriminate initial renal vascular effects of hypertension, despite the presence of a systemic vascular dysfunction, namely reduced FMD and increased PWV.

The evaluation of renal vasodilation to GTN by DRIN is able not only to confirm an impairment in renal microvasculature in type 2 diabetes, as already shown by RI, but also to detect a difference between hypertensive patients and both type 2 diabetic patients and controls. Therefore, the implementation of RI assessment by DRIN adds discriminating power to resting duplex evaluation of renal vascular resistances. We found no relationship between RI and DRIN in the group of type 2 diabetic patients, suggesting that a high baseline RI does not necessarily imply a reduced renal vasodilatory response, possibly reflecting functional, rather than structural, alterations. In patients with overt diabetic nephropathy, a reduced renal vasodilation was already demonstrated, using a high dose of GTN, to be able to influence systemic haemodynamics [17]. In comparison with that report, our work presents two important new elements: first, it suggests that DRIN could detect early renal impairment, even before the development of microalbuminuria; second, these results were obtained using subpressor doses of GTN, with the advantages of a better tolerability and the avoidance of systemic $\mathrm{BP}$ variation that may represent a relevant confounding factor in interpreting the results.

In contrast to DRIN, brachial artery response to GTN was similar in the three study groups. This apparent discrepancy is at least in part justified by the different characteristics of the two regions. Indeed, it is commonly 
accepted that renal RI, though measured at the level of the interlobar renal arteries, is an index of downstream renal microvascular impedance [12]; although a comparison between nitrate-induced changes in renal RI and other regions has never been performed, it was demonstrated that in type 2 diabetic patients skin microvascular reactivity [30], as well as forearm blood-flow changes [31] to sodium nitroprusside administration, are reduced, whereas brachial artery vasodilation to GTN is preserved [32]. Thus, it is conceivable that renal RI behaviour could be different from that of large arteries and similar to microcirculation.

To elucidate the determinants of DRIN and comparing them with RI, in order to comprehend the clinical significance of this new variable, a regression analysis was performed in the whole study group. First of all, both variables are more closely related to aortic than to brachial $\mathrm{BP}$ values, confirming that central $\mathrm{BP}$ reflects more accurately loading conditions of target organs [33]. In the univariate analysis, in addition to the classic cardiovascular risk factors, baseline RI was related to indices of systemic vascular damage. However, this relationship was lost in the multiple regression analysis. The main determinants of RI were, as expected, PP, as RI is an intrinsic measure of pulsatility, accompanied by serum glucose and creatinine. Thus, the link between RI and systemic vascular alterations appears to be mediated by the classic markers of cardiovascular and renal disease. At variance to RI, the independent predictors of DRIN were reactive hyperaemia and $\mathrm{PWV}$, together with $\mathrm{HbA}_{1 \mathrm{c}}$. A reduced vascular smooth muscle cell response or the presence of vascular remodelling in the microcirculation, as previously demonstrated in the forearm [31], could account for the observed reduction of DRIN in hypertensive and, particularly, in type 2 diabetic patients. Arterial stiffness could be related to renal vasodilatory capacity by two different mechanisms: both alterations could be manifestations of atherosclerosis in different districts [34]; otherwise, high PWV might lead to a heavier haemodynamic burden on the renal microcirculation, causing vascular remodelling and consequently reducing the vasodilatory capacity [35].

The mechanisms responsible for the onset of renal damage are conceivably different in the presence of hypertension or type 2 diabetes. PP is a strong predictor of DRIN in both conditions. Moreover, we show here that DRIN is independently related to arterial stiffness and wave reflection in hypertensive patients, suggesting a possible major role of haemodynamic load in determining early renal microvascular alterations in essential hypertension. On the contrary, in type 2 diabetic patients, DRIN is significantly related to $\mathrm{HbA}_{1 \mathrm{c}}$ and systolic BP. These observations support the importance of glucose and BP control in contrasting the development and progression of diabetic microvascular complications [36], and suggest that renal vasculature might be compromised even in the presence of more subtle glucose metabolism impairment, such as in the prediabetic condition, where systemic vascular dysfunction and increased arterial stiffness are already present [18].

In the present study, performed in normoalbuminuric individuals, neither RI nor DRIN correlated with UACR. Previous studies, including normo-, micro- and macroalbuminuric hypertensive patients, found a significant relationship between resting RI and microalbuminuria [13, 37]. Similarly, a study in type 2 diabetic patients showed that RI was associated with UACR only in albuminuric patients [14]. This could be related to the fact that an increase in both RI and UACR could be the expression of a more advanced phase of renal disease. It is also of note that, though still within the normoalbuminuric range, UACR levels are significantly higher in type 2 diabetic individuals than in the other two groups, and this is clinically relevant as the higher the albumin excretion, the worse the cardiovascular outcome [38]. Despite that, we found no correlation between UACR and DRIN or RI in the overall population or in the diabetic subgroup. This observation suggests, although only at a merely speculative level, that albuminuria and DRIN could be hallmarks of different mechanisms of renal damage, the former being more closely related to an altered glomerular permeability, with the latter to a more typical vascular damage.

Finally, a plausible common mechanism for impaired systemic and renal vascular function is represented by oxidative stress. In both groups of patients, the present study confirms the presence of such an alteration, as nitrotyrosine levels were higher in hypertensive patients, and even higher in patients with type 2 diabetes than in controls. Moreover, this systemic marker of oxidative stress correlates with RI, with the impairment of endotheliumdependent vasodilation and with aortic stiffness, although not independently affecting renal vascular variables in multivariate analysis.

In conclusion, the present study, although limited by the relatively small cohort of patients, supports the role of DRIN, a novel measure obtained by a widely available lowcost technique, as an early detector of renal vascular alteration in the presence of type 2 diabetes and hypertension, irrespective of the underlying mechanisms involved. The use of a vasodilator to unmask renal subclinical alterations is driven by the widespread use of challenges in other vascular areas and organs applied to different techniques, such as FMD for conduit large arteries and stress echocardiography for the myocardium. Further longitudinal studies are required in order to assess the clinical and prognostic value of an impaired drug-induced renal vasodilation; however, the demonstration of an impaired DRIN in patients with recent onset of the two 
main causes of chronic renal disease, along with the significant correlation of DRIN with other systemic markers of vascular dysfunction, supports its potential use as a clinical tool.

Duality of interest The authors declare that there is no duality of interest associated with this manuscript.

\section{References}

1. Mancia G, De Backer G, Dominiczak A et al (2007) 2007 Guidelines for the management of arterial hypertension: the Task Force for the Management of Arterial Hypertension of the European Society of Hypertension (ESH) and of the European Society of Cardiology (ESC). J Hypertens 25:1105-1187

2. Laurent S, Cockcroft J, Van Bortel L et al (2006) Expert consensus document on arterial stiffness: methodological issues and clinical applications. Eur Heart J 27:2588-2605

3. Virdis A, Ghiadoni L, Versari D, Giannarelli C, Salvetti A, Taddei S (2008) Endothelial function assessment in complicated hypertension. Curr Pharm Des 14:1761-1770

4. Ghiadoni L, Bruno RM, Stea F, Virdis A, Taddei S (2009) Central blood pressure, arterial stiffness, and wave reflection: new targets of treatment in essential hypertension. Curr Hypertens Rep $11: 190-196$

5. de Zeeuw D (2007) Albuminuria: a target for treatment of type 2 diabetic nephropathy. Semin Nephrol 27:172-181

6. Bakker SJ, Gansevoort RT, de Zeeuw D (2009) Albuminuria: what can we expect from the determination of nonimmunoreactive albumin? Curr Hypertens Rep 11:111-117

7. Futrakul N, Sridama V, Futrakul P (2009) Microalbuminuriaa biomarker of renal microvascular disease. Ren Fail 31:140143

8. Ruilope LM, Segura J (2009) Kidney protection: a key target in the management of patients with diabetes. J Hypertens 27(Suppl 1):S15-S18

9. Weir MR (2009) Hypertension and the kidney: perspectives on the relationship of kidney disease and cardiovascular disease. Clin J Am Soc Nephrol 4:2045-2050

10. MacIsaac RJ, Tsalamandris C, Panagiotopoulos S, Smith TJ, McNeil KJ, Jerums G (2004) Nonalbuminuric renal insufficiency in type 2 diabetes. Diabetes Care 27:195-200

11. Ikee R, Kobayashi S, Hemmi N et al (2005) Correlation between the resistive index by Doppler ultrasound and kidney function and histology. Am J Kidney Dis 46:603-609

12. Krumme B (2006) Renal Doppler sonography — update in clinical nephrology. Nephron Clin Pract 103:c24-c28

13. Derchi LE, Leoncini G, Parodi D et al (2005) Mild renal dysfunction and renal vascular resistance in primary hypertension. Am J Hypertens 18:966-971

14. Hamano K, Nitta A, Ohtake T, Kobayashi S (2008) Associations of renal vascular resistance with albuminuria and other macroangiopathy in type 2 diabetic patients. Diabetes Care 31:18531857

15. Radermacher J, Chavan A, Bleck J et al (2001) Use of Doppler ultrasonography to predict the outcome of therapy for renal-artery stenosis. N Engl J Med 344:410-417

16. Platt JF, Ellis JH, Rubin JM (1991) Examination of native kidneys with duplex Doppler ultrasound. Semin Ultrasound CT MR $12: 308-318$

17. Frauchiger B, Nussbaumer P, Hugentobler M, Staub D (2000) Duplex sonographic registration of age and diabetes-related loss of renal vasodilatory response to nitroglycerine. Nephrol Dial Transplant 15:827-832

18. Ghiadoni L, Penno G, Giannarelli C et al (2008) Metabolic syndrome and vascular alterations in normotensive subjects at risk of diabetes mellitus. Hypertension 51:440-445

19. Levey AS, Stevens LA, Schmid CH et al (2009) A new equation to estimate glomerular filtration rate. Ann Intern Med 150:604612

20. Ghiadoni L, Huang Y, Magagna A, Buralli S, Taddei S, Salvetti A (2001) Effect of acute blood pressure reduction on endothelial function in the brachial artery of patients with essential hypertension. J Hypertens 19:547-551

21. Gemignani V, Bianchini E, Faita F et al (2008) Ultrasound measurement of the brachial artery flow-mediated dilation without ECG gating. Ultrasound Med Biol 34:385-391

22. Plantinga Y, Ghiadoni L, Magagna A et al (2007) Supplementation with vitamins $\mathrm{C}$ and $\mathrm{E}$ improves arterial stiffness and endothelial function in essential hypertensive patients. Am J Hypertens 20:392397

23. Jerums G, Panagiotopoulos S, Premaratne E, MacIsaac RJ (2009) Integrating albuminuria and GFR in the assessment of diabetic nephropathy. Nat Rev Nephrol 5:397-406

24. Keogan MT, Kliewer MA, Hertzberg BS, DeLong DM, Tupler RH, Carroll BA (1996) Renal resistive indexes: variability in Doppler US measurement in a healthy population. Radiology 199:165-169

25. Platt JF, Ellis JH, Rubin JM (1991) Renal transplant pyelocaliectasis: role of duplex Doppler US in evaluation. Radiology 179:425-428

26. Platt JF, Marn CS, Baliga PK, Ellis JH, Rubin JM, Merion RM (1992) Renal dysfunction in hepatic disease: early identification with renal duplex Doppler US in patients who undergo liver transplantation. Radiology 183:801-806

27. Florczak E, Januszewicz M, Januszewicz A et al (2009) Relationship between renal resistive index and early target organ damage in patients with never-treated essential hypertension. Blood Press 18:55-61

28. Ishimura E, Nishizawa Y, Kawagishi T et al (1997) Intrarenal hemodynamic abnormalities in diabetic nephropathy measured by duplex Doppler sonography. Kidney Int 51:19201927

29. Pontremoli R, Viazzi F, Martinoli $C$ et al (1999) Increased renal resistive index in patients with essential hypertension: a marker of target organ damage. Nephrol Dial Transplant 14: 360-365

30. Lim SC, Caballero AE, Smakowski P, LoGerfo FW, Horton ES, Veves A (1999) Soluble intercellular adhesion molecule, vascular cell adhesion molecule, and impaired microvascular reactivity are early markers of vasculopathy in type 2 diabetic individuals without microalbuminuria. Diabetes Care 22:18651870

31. McVeigh GE, Brennan GM, Johnston GD et al (1992) Impaired endothelium-dependent and independent vasodilation in patients with type 2 (non-insulin-dependent) diabetes mellitus. Diabetologia 35:771-776

32. Henry RM, Ferreira I, Kostense PJ et al (2004) Type 2 diabetes is associated with impaired endothelium-dependent, flow-mediated dilation, but impaired glucose metabolism is not: the Hoorn Study. Atherosclerosis 174:49-56

33. Roman MJ, Devereux RB, Kizer JR et al (2007) Central pressure more strongly relates to vascular disease and outcome than does brachial pressure: the Strong Heart Study. Hypertension 50:197203

34. Ohta Y, Fujii K, Arima $\mathrm{H}$ et al (2005) Increased renal resistive index in atherosclerosis and diabetic nephropathy assessed by Doppler sonography. J Hypertens 23:1905-1911 
35. Safar ME, Lacolley P (2007) Disturbance of macro- and microcirculation: relations with pulse pressure and cardiac organ damage. Am J Physiol Heart Circ Physiol 293:H1-H7

36. Zoungas S, de Galan BE, Ninomiya T et al (2009) Combined effects of routine blood pressure lowering and intensive glucose control on macrovascular and microvascular outcomes in patients with type 2 diabetes: new results from the ADVANCE trial. Diabetes Care 32:2068-2074
37. Leoncini G, Martinoli C, Viazzi F et al (2002) Changes in renal resistive index and urinary albumin excretion in hypertensive patients under long-term treatment with lisinopril or nifedipine GITS. Nephron 90:169-173

38. Arnlov J, Evans JC, Meigs JB et al (2005) Low-grade albuminuria and incidence of cardiovascular disease events in nonhypertensive and nondiabetic individuals: the Framingham Heart Study. Circulation 112:969-975 